\title{
CAUCHY PROBLEMS FOR SEMI-LINEAR HYPERBOLIC SYSTEMS WITH THEIR APPLICATIONS
}

\section{E. E. NDIYO AND E. UKEJE}

(Received 01, April 2010; Revision Accepted 17, May 2010)

\begin{abstract}
In this paper, the solution to semi-linear hyperbolic systems of the form $A(x, t) u_{t}+B(x, t) u_{x}+c(x, t) u=f(x, t, u)$ in $\Omega x(0, \infty)$ with $u(x, 0)=\phi(x)$ is given. We apply this solution approached to establish the solution representation for any isentropic fluid flow.
\end{abstract}

KEYWORDS: Hyperbolic Systems, Characteristic curves, eigenvalues, eigenvectors, isentropic fluid.

\section{$1.0 \quad$ INTRODUCTION}

Cauchy problems arose naturally from physical phenomena and are of great interest. Different scholars like Lee-Datsin and Yu-wen Tzu (1981), Lagan (1976), Kong and Zhang (1996) had put forward various approaches in order to obtain a better result, by relaxing certain restrictions encountered in earlier results. In all, the characteristics concept appears to be the best and accepted method.

Nevertheless, the work is not complete given the varying physical principles where these Cauchy problems can be applied. The motion of compressible fluids offers instructive and significant illustrations of these characteristic concepts. Various forms expressing their flow exist depending on the space and media, whether in two dimensional space or spherically symmetric flow.

We have as an example the equations

$$
\begin{aligned}
& \rho u_{x}+u \rho_{x}+\rho_{t}=-\frac{2 \rho u}{x} \\
& \rho u u_{x}+\rho u_{t}+c^{2} \rho_{x}=0
\end{aligned}
$$

Moreso, considering Gas dynamics, either in Lagrangian coordinates or Eulerian coordinates, we have cases where the spatial variables will represent fixed points in space and in another instance, fixed particles.(Courant and Hilbert(1962),Michael and Robert(1993)). Thus, researches are inconclusive regarding any equation which may likely vary or be used to represent different phenomena like in water waves. In our earlier publication (Ndiyo 2005), we gave estimate solution to semi-linear hyperbolic systems in a generalized (weak) sense. In this paper, we consider in its generality the Cauchy problem of second order semi-linear hyperbolic equation of the form

$$
\begin{gathered}
A(x, t) u_{t t}+2 B(x, t) u_{t x}+c(x, t) u_{x x}+D(x, t) u_{t}+E(x, t) u_{x}=F(x, t, u) \\
u(x, 0)=\phi(x), u_{t}(x, 0)=\psi(x)
\end{gathered}
$$

We reduced equation 1.3 - 1.4 into a first order system and obtained its eigenvalues and left eigenvectors, from which we established its solution representation. We then illustrate this solution using an isentropic fluid flow equation.

\subsection{System of First Order and Solution Representation}

Given the equation $1.3-1.4$, let $p(x, t)$ be $u_{t}$ and $q(x, t)=u_{x}$. We differentiate and substitute in 1.3 to obtain

$$
A(x, t) p_{t}+B(x, t) p_{x}+B(x, t) q_{t}+C(x, t) q_{x}+D(x, t) p+E(x, t) q=F(x, t, u)
$$

and

$$
q_{t}-p_{x}=0
$$

by the continuity of mixed derivatives.

Equations (2.1) and (2.2) becomes a system written

E. E. Ndiyo, Dept. Of Maths/Stats/Computer Science, University Of Uyo, Uyo, Nigeria.

E. Ukeje, Dept. Of Maths/Stats/Comp. Science, Michael Okpara University of Agriculture, Umudike, Nigeria. 


$$
\begin{aligned}
& \left(\begin{array}{ll}
A & B \\
0 & 1
\end{array}\right)\left(\begin{array}{l}
p \\
q
\end{array}\right)_{t}+\left(\begin{array}{cc}
B & C \\
-1 & 0
\end{array}\right)\left(\begin{array}{l}
p \\
q
\end{array}\right)_{x}+\left(\begin{array}{cc}
D & E \\
0 & 0
\end{array}\right)\left(\begin{array}{l}
p \\
q
\end{array}\right)=\left(\begin{array}{l}
F \\
0
\end{array}\right) \\
& p(x, 0)=\phi(x), q(x, 0)=\psi(x)
\end{aligned}
$$

The matrix $k=\left(\begin{array}{cc}A & B \\ 0 & 1\end{array}\right)$ is non-singular and for $A \neq 0$ will have its inverse as $k^{-1}=\left(\begin{array}{cc}1 / A & -B / A \\ 0 & 1\end{array}\right)$

The system (2.3) and (2.4) is reduced to $\quad V_{t}+m V_{x}+N v=f$

where $\quad m=\left(\begin{array}{cc}2 B / A & C / A \\ -1 & 0\end{array}\right), N=\left(\begin{array}{cc}D / A & E / A \\ 0 & 0\end{array}\right)$

and

$$
f=\left(\begin{array}{c}
F(x, t, u) \\
0
\end{array}\right)
$$

The eigenvalues of the principal part ' $m$ ' will be

$$
\lambda_{i}=\frac{B \pm \sqrt{B^{2}-A C}}{A} \quad i=1,2 .
$$

The resulting eigenvectors matrix which are functions of $(x, t)$ is

$$
\begin{aligned}
& S=\left(\begin{array}{ll}
\frac{-\lambda_{1}}{\sqrt{1+\lambda_{1}^{2}}} & \frac{-\lambda_{2}}{\sqrt{1+\lambda_{2}^{2}}} \\
\frac{1}{\sqrt{1+\lambda_{1}^{2}}} & \frac{1}{\sqrt{1+\lambda_{2}^{2}}}
\end{array}\right) \\
& S^{-1}=\frac{\sqrt{\left(1+\lambda_{1}^{2}\right)\left(1+\lambda_{2}\right)^{2}}}{\lambda_{2}-\lambda_{1}}\left(\begin{array}{cc}
\frac{1}{\sqrt{1+\lambda_{1}^{2}}} & \frac{\lambda_{2}}{\sqrt{1+\lambda_{2}^{2}}} \\
\frac{-1}{\sqrt{1+\lambda_{1}^{2}}} & \frac{-\lambda_{1}}{\sqrt{1+\lambda_{1}^{2}}}
\end{array}\right)
\end{aligned}
$$

The matrix $\mathrm{m}$ in a diagonalised form

makes the system to become

$$
m=S \wedge S^{-1}, \wedge=\operatorname{diag}\left(\lambda_{1}, \lambda_{2}\right)
$$

$$
\left(\begin{array}{l}
p \\
q
\end{array}\right)_{t}+S \wedge S^{-1}\left(\begin{array}{l}
p \\
q
\end{array}\right)_{x}+\left(\begin{array}{cc}
D / A & E / A \\
0 & 0
\end{array}\right)\left(\begin{array}{l}
p \\
q
\end{array}\right)=\left(\begin{array}{c}
f(x, t, u) \\
0
\end{array}\right)
$$

Let $\quad\left(\begin{array}{l}r \\ h\end{array}\right)=S^{-1}\left(\begin{array}{l}p \\ q\end{array}\right)$, we have from (2.5).

$$
\begin{aligned}
\left(\begin{array}{l}
r \\
h
\end{array}\right)_{t}-S_{t}^{-1} S\left(\begin{array}{l}
r \\
h
\end{array}\right)+\wedge\left\{\left(\begin{array}{l}
r \\
h
\end{array}\right)_{x}-S_{x}^{-1} S\left(\begin{array}{l}
r \\
h
\end{array}\right)\right\} \\
+S^{-1}\left(\begin{array}{cc}
P / A & E \\
0 & 0
\end{array}\right) S\left(\begin{array}{l}
r \\
h
\end{array}\right)=S^{-1}\left(\begin{array}{c}
f(x, t, u) \\
0
\end{array}\right)
\end{aligned}
$$

Define

$$
Q=\left(\begin{array}{ll}
A_{1}^{*} & A_{2}^{*} \\
B_{1}^{*} & B_{2}^{*}
\end{array}\right)=S^{-1}\left(\begin{array}{cc}
D / A & E / A \\
0 & 0
\end{array}\right) S-S_{t}^{-1} S-A\left(S_{x}^{-1} S\right)
$$

we obtained the system of equations

$$
\left(\begin{array}{l}
r \\
h
\end{array}\right)_{t}+\wedge\left(\begin{array}{l}
r \\
h
\end{array}\right)_{x}+\left(\begin{array}{ll}
A_{1}^{*} & A_{2}^{*} \\
B_{1}^{*} & B_{2}^{*}
\end{array}\right)\left(\begin{array}{l}
r \\
h
\end{array}\right)=\left(\begin{array}{l}
\frac{C_{1}^{*} f(x, t, u)}{A} \\
\frac{C_{2}^{*} f(x, t, u)}{A}
\end{array}\right)
$$




$$
r(x, 0)=S^{-1} \phi(x) \text { and } h(x, 0)=S^{-1} \psi(x)
$$

Equation 2.8 and 2.9 becomes system of characteristics equations for the Cauchy problem $1.3-1.4$. Along each curves $\mathrm{c}_{1}$ and $\mathrm{c}_{2}$ corresponding to each eigenvalues $\lambda_{i}, i=1,2$ we get

$$
\begin{aligned}
& \frac{d r}{d t}+\lambda_{1} \frac{d r}{d x}+A_{1}^{*} r+A_{2} h=\frac{C_{1}^{*}}{A} f(x, t, u) \\
& \frac{d h}{d t}+\lambda_{2} \frac{d h}{d x}+B_{1}^{*} r+B_{2}^{*} h=\frac{C_{2}^{*}}{A} f(x, t, u)
\end{aligned}
$$

The solution representations are expressed by the corresponding integral taken over the characteristic curves with respect to $\mathrm{t}$ for $0 \leq t \leq \xi$ thus;

$$
\begin{aligned}
r(x, t)= & r(x, 0) e^{-\int^{\varepsilon} A_{1}^{*}(x, \tau) d \tau}-\int_{0}^{t}\left[A_{2}^{*}(x, \xi) e^{-\int^{\varepsilon} A_{1}^{*}(x, \tau) d \tau}\right] e^{-\int^{\varepsilon} A_{1}^{*}(x, \tau) d \tau} d \varepsilon \\
& +\int_{0}^{+}\left[\frac{C_{1}^{*}(x, \varepsilon)}{A(x, \varepsilon)} f(x, \xi, u) e^{-\int A_{1}^{*}(x, \tau) d \tau}\right] e^{-\int A_{1}^{*}(x, \tau) d \tau} d \varepsilon \\
h(x, t)= & h(x, 0) e^{-\int^{\varepsilon} B_{1}^{*}(x, \tau) d \tau}-\int_{0}^{t}\left[B_{2}^{*}(x, \eta)(x, \eta) e^{-\int^{\varepsilon} B_{1}^{*}(x, \tau) d \tau}\right] e^{-\int^{\varepsilon} B_{1}^{*}(x, \tau) d \tau} d \eta \\
& +\int_{0}^{+}\left[\frac{C_{2}^{*}(x, \eta)}{A(x, \eta)} f(x, \eta, u) e^{-\int^{\varepsilon} B_{1}^{*}(x, \tau) d \tau}\right] e^{-\int^{\varepsilon} B_{1}^{*}(x, \tau) d \tau} d \eta
\end{aligned}
$$

we eliminate $h(x, \varepsilon)$ and $r(x, \xi)$ from each of the solution representation as applicable to obtain

$$
\begin{aligned}
& r(x, t)= r(x, 0) e^{-\int^{\varepsilon} A_{1}^{*}(x, \tau) d \tau}+\int_{0}^{t}\left[\int_{-}^{\varepsilon} B_{1}^{*}(x, \eta) r(x, \eta) e^{-\int^{\varepsilon} B_{1}^{*}(x, \tau) d \tau} d \eta\right] A_{2}^{*}(x, \xi) \\
&-e^{-\int A_{1}^{*}(x, \tau) d \tau} d \xi+\int_{0}^{+}\left[\int_{0}^{\xi} \frac{C_{2}^{*}(x, \eta)}{A(x, \eta)} f(x, \eta, u) e^{-\int^{\xi} B_{1}^{*}(x, \tau) d \tau} d \eta\right] A_{1}^{*}(x, \xi) \\
& e^{-\int^{\varepsilon} A_{1}^{*}(x, \tau) d \tau} d \varepsilon+\int_{0}^{t} \frac{C_{1}^{*}(x, \varepsilon)}{A(x, \varepsilon)} f(x, \xi, u) e^{-\int_{0}^{\xi} B_{1}^{*}(x, \tau) d \tau} d \varepsilon \\
& h(x, t)=h(x, 0) e^{-\int B_{1}^{*}(x, \tau) d \tau}+\int_{0}^{t}\left[\int_{0}^{\varepsilon} A_{2}^{*}(x, \xi) h(x, \xi) e^{-\int A_{1}^{*}(x, \tau) d \tau} d \xi\right] B_{2}^{*}(x, \eta) \\
& e^{-\int B_{1}^{*}(x, \tau) d \tau} d \eta+\int_{0}^{t}\left[\int_{0}^{\xi} \frac{C_{1}^{*}(x, \xi)}{A(x, \xi)} f(x, \xi, u) e^{-\int_{t}^{\xi} A_{1}^{*}(x, \tau) d \tau}\right] B_{1}^{*}(x, \eta) \\
& e^{-\int^{\varepsilon} B_{2}^{*}(x, \tau) d \tau} d \eta+\int_{0}^{t} \frac{C_{2}^{*}(x, \eta)}{A(x, \eta)} f(x, \eta, u) e^{-\int^{\xi} B_{2}^{*}(x, \tau) d \tau} d \eta
\end{aligned}
$$

It has been shown earlier by Ndiyo-Ukeje (2004) that these solutions $r(x, t)$ and $h(x, t)$ does exist and are unique within the domain of dependency of the solution.

\subsection{Application}

Consider the equations

$$
\rho_{t}+(\rho u)_{x}=0
$$




$$
(\rho u)_{t}+\left(\rho u^{2}\right)_{x}+\rho_{x}=0
$$

where $\rho$ is the density and $\mathrm{u}$ the velocity of the fluid in the $\mathrm{x}$-direction.

The matrix form is

$$
\left(\begin{array}{ll}
1 & 0 \\
u & \rho
\end{array}\right)\left(\begin{array}{l}
\rho_{t} \\
u_{t}
\end{array}\right)+\left(\begin{array}{cc}
u & \rho \\
u^{2} & 2 \rho u
\end{array}\right)\left(\begin{array}{l}
\rho_{x} \\
u_{x}
\end{array}\right)=\left(\begin{array}{c}
0 \\
-\rho_{x}
\end{array}\right)
$$

represented as

$$
A \bar{v}_{t}+B \bar{v}_{x}=F
$$

where A is non-singular since $\rho \neq 0$ with inverse

$$
A^{-1}=\left(\begin{array}{cc}
1 & 0 \\
-u / \rho & 1 / \rho
\end{array}\right)
$$

Multiplying equation 3.3 by $A^{-1}$, we obtain

$$
\left(\begin{array}{l}
\rho_{t} \\
u_{t}
\end{array}\right)+\left(\begin{array}{ll}
u & \rho \\
0 & u
\end{array}\right)\left(\begin{array}{l}
\rho_{x} \\
u_{x}
\end{array}\right)=\left(\begin{array}{c}
0 \\
-\rho_{x} / \rho
\end{array}\right)
$$

having the eigenvalues as $\lambda_{1}=\lambda_{2}=u$.which is a case of multiplicity of eigenvalues.

The eigenvector matrix becomes

$$
\begin{aligned}
& S=\left(\begin{array}{cc}
1 & 0 \\
0 & -1
\end{array}\right) \\
& S^{-1}=\left(\begin{array}{cc}
1 & 0 \\
0 & -1
\end{array}\right)
\end{aligned}
$$

By writing the system in its diagonalised form, we get

$$
\begin{aligned}
& \left(\begin{array}{l}
\rho \\
u
\end{array}\right)_{t}+S \wedge S^{-1}\left(\begin{array}{l}
\rho \\
u
\end{array}\right)_{x}=\left(\begin{array}{c}
0 \\
-\rho_{x} / \rho
\end{array}\right) \\
& S^{-1}\left(\begin{array}{l}
\rho \\
u
\end{array}\right)_{t}+\wedge S^{-1}\left(\begin{array}{l}
\rho \\
u
\end{array}\right)_{x}=S^{-1}\left(\begin{array}{c}
0 \\
-\rho_{x} / \rho
\end{array}\right) \\
\Rightarrow \quad & \left(\begin{array}{cc}
1 & 0 \\
0 & -1
\end{array}\right)\left(\begin{array}{l}
\rho_{t} \\
u_{t}
\end{array}\right)+\wedge\left(\begin{array}{cc}
1 & 0 \\
0 & -0
\end{array}\right)\left(\begin{array}{l}
\rho_{x} \\
u_{x}
\end{array}\right)=\left(\begin{array}{cc}
1 & 0 \\
0 & -1
\end{array}\right)\left(\begin{array}{c}
0 \\
-\rho_{x} / \rho
\end{array}\right) \\
\Rightarrow \quad & \left(\begin{array}{l}
\rho_{t} \\
u_{t}
\end{array}\right)+\left(\begin{array}{ll}
u & 0 \\
0 & u
\end{array}\right)\left(\begin{array}{l}
\rho_{x} \\
u_{x}
\end{array}\right)=\left(\begin{array}{c}
0 \\
-\frac{\rho_{x}}{\rho}(x, t, u)
\end{array}\right)
\end{aligned}
$$

Indicating the component form along the characteristic curves, where

$$
\frac{d \rho}{d t}=0
$$

making $\rho=$ constant

then $\frac{d u}{d t}=-\frac{\rho_{x}}{\rho}(x, t, u)$

From the solution representation, we have that $Q \equiv 0$ from equation 2.7.

Hence

$$
u(x, t)=u(x, 0)+\int_{0}^{t}-\frac{\rho_{x}}{\rho}(x, \xi, u) d \xi
$$

Following the uniqueness theorem criteria by Lagan (1976),this solution does exist and it is unique if the integrand is Lipschitz continuous with respect to $u$. 


\section{REFERENCES}

Lee-Da-Tsin \& Yu-wen Tzu 1981. Boundary value problems for the first order quasi-linear hyperbolic systems and their applications. J. Differential Equations vol. 41:1-26.

Thomas J. L., 1976. Existence and Uniqueness of Solutions to Initial-Boundary value problems for semi-linear hyperbolic systems in two independent variables.

J. Differential Equations vol. 22:74-88.

Kong De-xing \& Zhang Wei-guo 1996. Cauchy Problem for Quasi-linear hyperbolic systems with higher order Dissipative Terms. ICTP - IC/96/56. Miramane, Trieste, Italy.

Courant-Hilbert 1962. Methods of Mathematical Physics Vol. 2; New York Inter science Publishers John Wiley and Sons.

Michael Renardy \& Robert C. R.,1993. An Introduction to Partial Differential Equations . New York, Springer-Verlag, Berlin.

Ndiyo, E. E., 2005. Estimate solution to first order semi-linear hyperbolic partial differential equation. Global Journal of Mathematical Science vol. $4.1 \& 2 ; 11-17$.

Ndiyo, E. E. and Ukeje E., 2004. Existence and Uniqueness of Solution of First Order Quasilinear Hyperbolic Systems in Two-Independent variables using fixed point theorems. Global Journal of Math. Sciences vol. 3 No. 1:5-10. 